\title{
The Implications of an Idealized Large-Scale Circulation for Mechanical Work Done by Tropical Convection
}

\author{
Jan A. Kamieniecki, MaArten H. P. Ambaum, and Robert S. Plant \\ Department of Meteorology, University of Reading, Reading, United Kingdom
}

STEVEN J. WoOLNOUGH

Climate, National Centre for Atmospheric Science, and Department of Meteorology, University of Reading, Reading, United Kingdom

(Manuscript received 19 October 2017, in final form 9 May 2018)

\begin{abstract}
A thermodynamic analysis is presented of an overturning circulation simulated by two cloud-resolving models, coupled by a weak temperature gradient parameterization. Taken together, they represent two separated regions over different sea surface temperatures, and the coupling represents an idealized largescale circulation such as the Walker circulation. It is demonstrated that a thermodynamic budget linking net heat input to the generation of mechanical energy can be partitioned into contributions from the large-scale interaction between the two regions, as represented by the weak temperature gradient approximation, and from convective motions in the active warm region and the suppressed cool region. Model results imply that such thermodynamic diagnostics for the aggregate system are barely affected by the strength of the coupling, even its introduction, or by the SST contrast between the regions. This indicates that the weak temperature gradient parameterization does not introduce anomalous thermodynamic behavior. We find that the vertical kinetic energy associated with the large-scale circulation is more than three orders of magnitude smaller than the typical vertical kinetic energy in each region. However, even with very weak coupling circulations, the contrast between the thermodynamic budget terms for the suppressed and active regions is strong and is relatively insensitive to the degree of the coupling. Additionally, scaling arguments are developed for the relative values of the terms in the mechanical energy budget.
\end{abstract}

\section{Introduction}

The representation of the interactions between largescale tropical circulations and local convective processes is an issue of fundamental importance to the simulation of tropical circulations. Computational constraints mean that models that explicitly simulate both convection and largescale motion over domains of appropriate size can rarely be used. Hence, a strategy, often adopted in the development of convection parameterizations for the tropics for GCMs, is to carry out studies using cloud-resolving models (CRMs) that explicitly model convection in conjunction with a parameterization of the large-scale dynamics that is influenced by local conditions. The weak temperature gradient (WTG) approximation (Sobel and Bretherton 2000; Raymond and Zeng 2005) is one such parameterization.

Corresponding author: Jan A. Kamieniecki, j.a.kamieniecki@ pgr.reading.ac.uk
The atmospheric circulation can be analyzed from the perspective of a generalized Carnot heat engine that converts a temperature difference between two reservoirs into mechanical energy [e.g., Emanuel (1986) for the case of tropical cyclones]. It has been shown by Pauluis (2011) that the impact of the hydrological cycle is to reduce the efficiency of such a conversion relative to the generalized Carnot maximum. This paper addresses convective and large-scale circulations as heat engines, unpacking how efficient they are at transforming potential work into kinetic energy dissipation and water lifting, in the face of the thermodynamic penalties associated with irreversible phase transitions in a moist atmosphere. Hitherto, similar analyses have not been applied in a context where the influence of large-scale circulations on local convection is modeled separately. Such an approach has the potential not only to clarify the consequences of different large-scale parameterization approaches and to identify constraints on them but also to shed light on the nature of the interaction 
between the large-scale and the local dynamics. In particular, we examine whether the mechanical energy budget is well behaved when two convecting regions are coupled by a parameterized large-scale circulation.

The WTG approximation was first suggested by Sobel and Bretherton (2000) and subsequently modified by introducing a short relaxation time (e.g., Raymond and Zeng 2005). Alternative methods such as the damped gravity wave (DGW) approach (Kuang 2008; Romps 2012), a spectral variant of the WTG (Herman and Raymond 2014), and others have been proposed. In this paper, we focus on the weak temperature gradient approximation, but work on the DGW approach is ongoing. Preliminary analysis suggests that the DGW approach would give similar results to those presented in this paper.

A recent project has compared the results produced by different implementations of some of these methods (Daleu et al. 2015b, 2016), suggesting that the WTG approach is more prone to multiple equilibria than the DGW approach and that the latter produces smoother large-scale vertical velocities. One of the advantages of the WTG approach is that its simplicity permits an analytical approach, and for that reason, it is used here.

A common formulation of the WTG approximation assumes that a large-scale vertical wind is prescribed so as to contribute to the elimination of horizontal temperature contrasts. This paper adopts a typical definition, where the prescribed vertical large-scale wind $w_{\mathrm{LS}}$ is

$$
w_{\mathrm{LS}} \frac{\partial \theta_{r}}{\partial z}=\frac{\theta^{\prime}}{\tau}
$$

where $\theta_{r}$ represents a reference profile for potential temperature, $\theta^{\prime}$ a local potential temperature anomaly, $\tau$ a relaxation time scale, and $z$ the height above mean sea level; $w_{\mathrm{LS}}$ is usually interpolated to zero at the surface from the predicted value at the top of the planetary boundary layer and a minimum value for the static stability imposed. The time scale $\tau$ represents the strength of the coupling between regions, and typical values of 2-3 $\mathrm{h}$ can be justified as the spatial scale of the system being modeled $(\sim 500 \mathrm{~km})$ divided by the speed of the fastest internal gravity waves $\left(\sim 50 \mathrm{~m} \mathrm{~s}^{-1}\right)$.

This circulation can be superimposed on the normal convective motions representing an idealized large-scale circulation that provides a linkage between two regions. Most studies have used a reference-column approach, whereby the large-scale circulation is determined by temperature differences between the area modeled and an assumed environmental profile; more recently, Daleu et al. $(2012,2015 a)$ extended this approach to two coupled regions, which enables a more explicit representation of the consequences of the large-scale coupling mechanism. The reference column approach implies an infinite reservoir of energy and entropy, whereas by using coupled regions, it is possible to analyze the mechanical energy budget of a closed system, separately attributing the contribution of the large-scale circulation described above and of the residual convective motions in the two regions.

The approach to the diagnosis of the mechanical work done by convection adopted in this paper broadly follows the isentropic perspective introduced by Pauluis and Mrowiec (2013) and Pauluis (2016). The conditional average of the vertical mass flux of air parcels, $\langle\rho w\rangle$, where $\rho$ is the density of dry air and $w$ is vertical velocity, is calculated in $\left(z, \theta_{e}\right)$ space, $\theta_{e}$ being the equivalent potential temperature. This is illustrated in Fig. 1a. This conditionally averaged vertical mass flux can be used to derive a streamfunction $\Psi$ (Fig. 1b):

$$
\Psi\left(z, \theta_{e}\right)=\int_{0}^{\theta_{e}}\langle\rho w\rangle\left(z, \theta_{e}^{\prime}\right) d \theta_{e}^{\prime} .
$$

This describes mass flows in $\left(z, \theta_{e}\right)$ space and can be regarded as a projection onto thermodynamic variables of the overturning circulation. It may be treated as a streamfunction providing divergent flows in that space do not exist. Likewise, conditional averages in $\left(z, \theta_{e}\right)$ space of variables such as temperature $T$, vapor mixing ratio $r_{v}$, liquid water mixing ratio $r_{l}$, ice mixing ratio $r_{i}$, entropy $S$, and buoyancy $B$ can be obtained. See Pauluis and Mrowiec (2013) for a further discussion of the merits of this approach.

Pauluis (2016) derives an approximate expression that relates components of work produced by a closed contour of the streamfunction, which can be expressed as

$$
\underbrace{\oint T d S}_{W_{T d S}}-\underbrace{\sum_{w=v, l, i} \oint\left(-\mathscr{G}_{w}\right) d r_{w}}_{\Delta \mathscr{G}} \approx \underbrace{\oint B d z}_{W_{b}}+\underbrace{g \oint r_{T} d z}_{W_{p}},
$$

where $r_{T}=r_{v}+r_{l}+r_{i}$ is the total water mixing ratio, $g$ represents the acceleration due to gravity, and $\mathscr{S}_{w}$ the specific Gibbs free energy for the various phases of water represented in the model; $W_{T d S}$, the first item on the left-hand side of this equation, can be interpreted as the work that would be done by a generalized Carnot engine with the same path in $(T, S)$ coordinates. The second term, $\Delta \mathscr{G}$, referred to as the "Gibbs penalty," is the lost part of that work that is needed to evaporate liquid water at below saturation into the system in order to maintain the mean humidity profile against the hydrological cycle and is discussed in detail in 


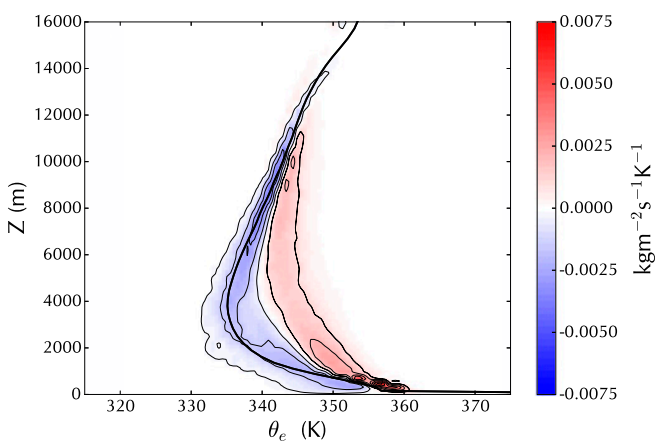

(a) Isentropic distribution of the vertical mass flux

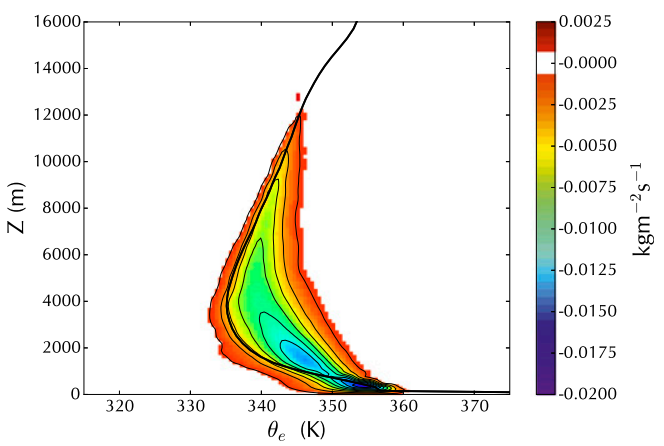

(b) Isentropic stream function

FIG. 1. (a) Isentropic distribution and (b) streamfunction of the vertical mass flux $\langle\rho w\rangle$ for a single uncoupled region at RCE over an SST of $302.7 \mathrm{~K}$. The solid line represents the mean profile of the equivalent potential temperature $\theta_{e}$.

Pauluis and Held (2002a,b) and Pauluis (2011). The first term on the right-hand side, $W_{b}$, is the generation of kinetic energy by resolved motions in the model and the second, $W_{p}$, the increase in geopotential energy of the water content lifted by convection. Ultimately, the buoyancy term $W_{b}$ generates kinetic motion of air, which is dissipated through turbulent processes mostly near the surface, and the moisture elevator term $W_{p}$ generates potential energy in hydrometeors, which is subsequently dissipated through friction during precipitation (Pauluis et al. 2000). The Gibbs penalty term does not correspond to external energy transiting through the system but serves to limit the work available for conversion into mechanical energy. In this sense, the Gibbs penalty is the part of the entropy budget that needs to be expended to maintain the composition changes in the hydrological cycle and that therefore cannot be used to produce mechanical work.

The terms in this equation can be represented by thermodynamic diagrams of streamfunction contours in the appropriate spaces, for example, that for $W_{T d S}$ in $(S, T)$ space. A weighted total of integrals along equally spaced contours of $\Psi$ is used to calculate values of the terms in Eq. (3) for the entire system. Figure 2 shows contours for the most significant terms in the case of a single region in radiative-convective equilibrium (RCE) for the model configuration described below.

This paper applies the diagnostic approach outlined above to unpack the generation of mechanical work by a modeled overturning circulation superimposed on convective processes. In particular, it analyses the relationship between the strength of WTG coupling and the strength of the circulation, partitioned into contributions from the large-scale circulation and convective circulations in relatively cool/dry and warm/moist regions, in order to deepen understanding of how the large-scale circulation can suppress or enhance convection. The impact of varying the SST difference between the regions is also analyzed.

The paper is organized as follows. Section 2 explains how an isentropic analysis can be applied to two coupled regions and also suggests a refinement that improves the accuracy of the decomposition for an anelastic model. Section 3 briefly describes the CRM used and presents numerical results for the mechanical energy budget, partitioned into a large-scale circulation and convective localized flows for varying strengths of coupling as well as further results for varying SST differences between the two regions. Section 4 develops an analytical expression for the generation of kinetic energy by the WTG coupling, which is seen to be consistent with results obtained from the CRM. Scaling arguments are also developed for comparison with other results obtained by the CRM. The implications of this study are discussed and some conclusions are drawn in section 5.

\section{Isentropic diagnostic framework}

\section{a. General approach}

As in Pauluis (2016), entropy is expressed as specific entropy per unit mass of dry air:

$$
s=s_{d}+r_{v} s_{v}+r_{l} s_{l}+r_{i} s_{i},
$$

where $s_{d}$ is the specific entropy of dry air and $s_{w}$ for $w=v, l, i$ are the specific entropies of the three phases of water. To achieve a one-to-one relationship between equivalent potential temperature and this specific entropy, the former is defined as

$$
\theta_{e}=T_{0} \ln \frac{s}{c_{p}}
$$




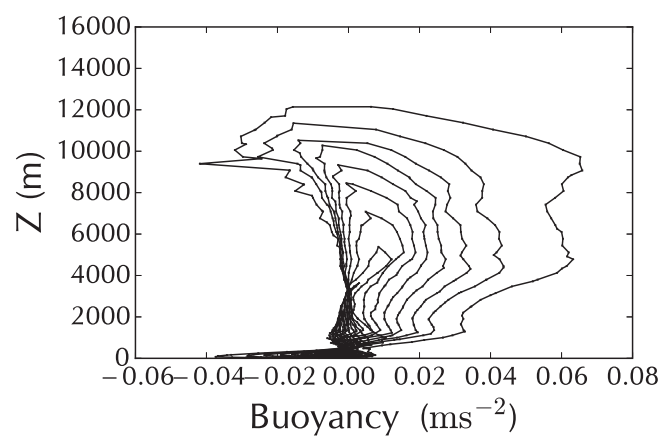

(a) $B-z$

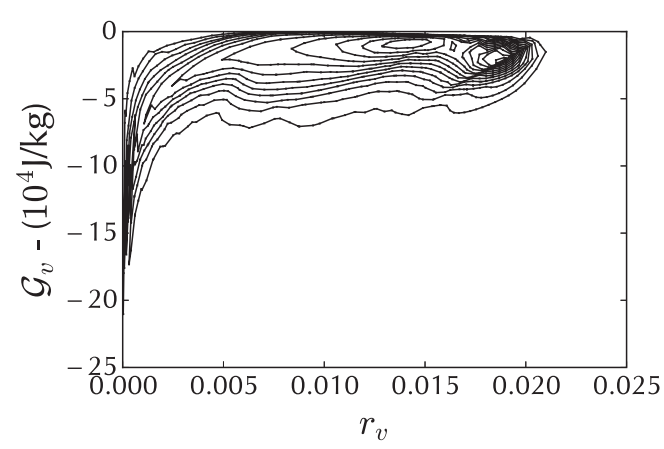

(c) $r_{v}-\mathscr{G}^{*}$

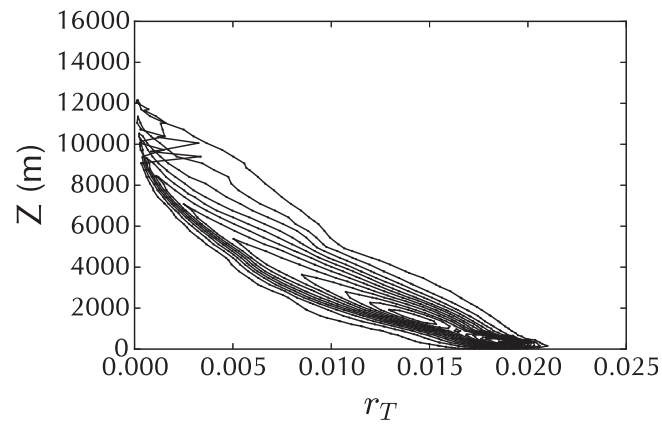

(b) $r_{T}-z$

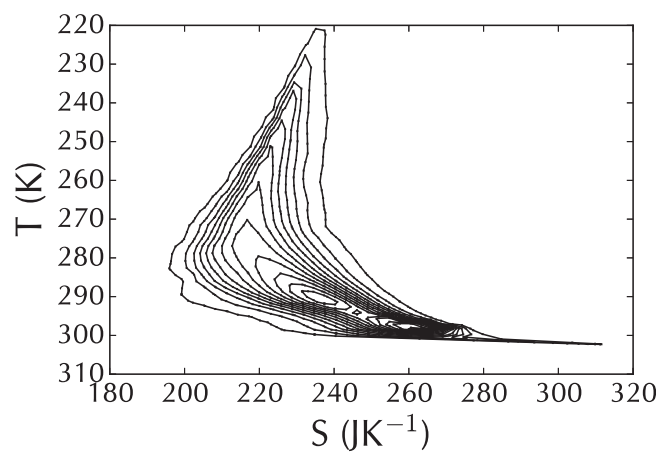

(d) $S-T$

FIG. 2. Thermodynamic diagrams for the main terms in Eq. (3) for a single uncoupled region at RCE over a surface at temperature $302.7 \mathrm{~K}$ showing 15 equally spaced contours of the streamfunction. (Numerical values elsewhere in this paper were calculated using 60 contours.)

where $T_{0}$ is the temperature at the triple point of water and $c_{p}$ is the heat capacity of dry air at constant pressure.

Pauluis (2016) uses a weighted average of equally spaced contours of the streamfunction over the region where $\Psi<0$ to calculate the components of overall work throughput of the system as expressed in Eq. (3). Following that approach, these integrals are calculated here along contours of the streamfunction in $\left(z, \theta_{e}\right)$ space, using the conditionally averaged values of the variables required for the calculation [such as $(T, S)$ for $\left.W_{T d S}\right]$ of the points defining the trajectory, rather than seeking first to determine a streamfunction in $(T, S)$ space and then to calculate contour integrals in that space. The same results can also be calculated using appropriate surface integrals of the streamfunction; for example, the first term on the left-hand side of Eq. (3) becomes $\iint \Psi d S d T$ over the same domain. Although the results shown in this paper are calculated using contours, the surface integral approach is important in justifying the partitioning approach described below.

\section{b. Extension to two-region case}

This paper applies the decomposition of work done discussed above to a two-region system, where the regions are coupled by a large-scale circulation consisting of vertical winds specified by the WTG approximation. The cooler region is identified by a subscript 1 and represents a fraction $(1-\varepsilon)$ of the total area and the warmer region (subscript 2) a fraction $\varepsilon$ (all the numerical results shown in this paper are based on $\varepsilon=0.5$ ). When a temperature difference between the surfaces underlying the two regions gives rise to a large-scale circulation, there will be mass transfers between the two regions, and the contours of the integral of the vertical mass flux [as in Eq. (2)] in each region will not be closed, making it impossible to estimate the components of Eq. (3) within each region using either contour or surface integrals (the latter requiring a domain bounded by a constant value of the streamfunction).

One could, of course, merge the two regions prior to performing any analysis, but this introduces significant error as the dynamics are generally influenced by local rather than merged values of fields (e.g., buoyancy for $W_{b}=\oint B d z$ ), and such a method will not permit a partitioning of the components of work done to localized features in each region and to the large-scale circulation.

Instead, one can identify and separate out the vertical large-scale velocities $w_{\mathrm{LS}, 1}(z), w_{\mathrm{LS}, 2}(z)$ [obtained from 


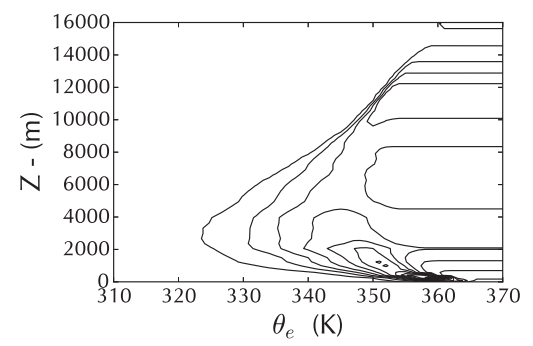

(a) Cooler column

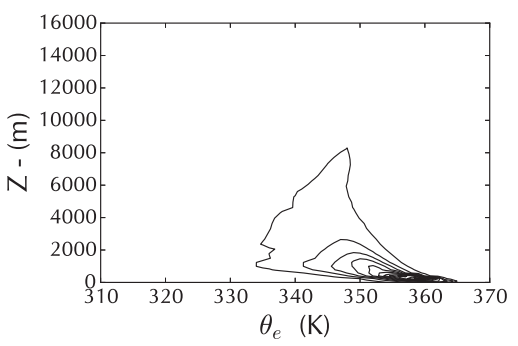

(d) Cooler column - local

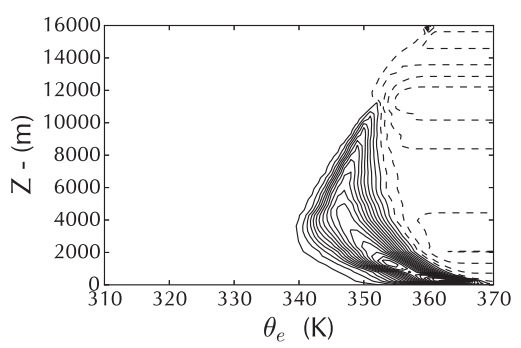

(b) Warmer column

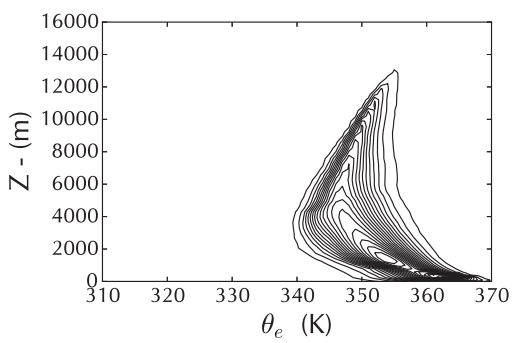

(e) Warmer column - local

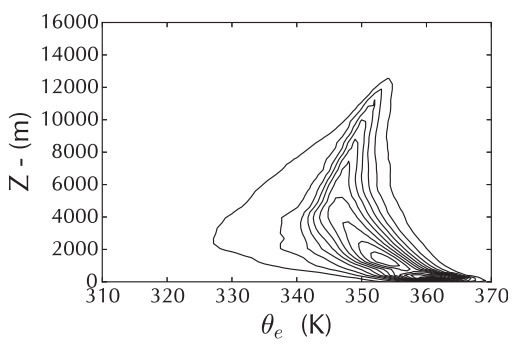

(c) Total system

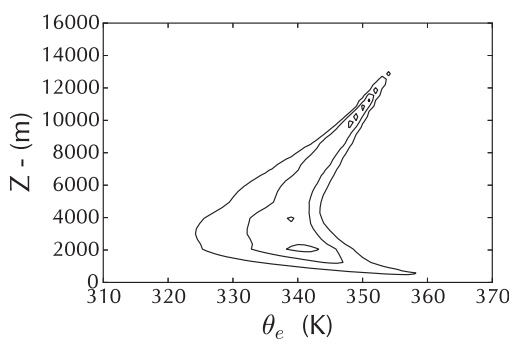

(f) Large-scale circulation

FIG. 3. (a),(b) Contours of the integrated vertical mass flux for each of the two regions analyzed in isolation. The regions are run to RCE and are coupled with $\tau=2 \mathrm{~h}$. Note that the contours are not closed and so cannot be represented by a streamfunction. (c) The streamfunction of the total system. (f) Once the large-scale circulation is treated separately, (d),(e) the residual "convective" circulations in the two regions exhibit closed contours. The same values for the contours are used in each of the plots; here, solid lines indicate negative values and dashed lines positive values.

the values of $\Psi\left(z, \theta_{e}\right)$ as $\theta_{e} \rightarrow \infty$ and the dry-airmass distribution in each region]. The circulations calculated using Eq. (2) for convective local velocities $w_{i}^{*}=w_{i}-w_{\mathrm{LS}, i}$ for $i=1,2$ in each region will now be closed, as will that in a notional third "region" that consists of the total of the large-scale circulation within the two regions, weighted by their relative areas, since $(1-\varepsilon) w_{\mathrm{LS}, 1}+\varepsilon w_{\mathrm{LS}, 2}=0$. These streamfunctions are additive by construction:

$$
\Psi_{1}+\Psi_{2}=\Psi_{1}^{*}+\Psi_{2}^{*}+\Psi_{\mathrm{LS}}
$$

where $\Psi_{1,2}$ are the observed circulations in each region, $\Psi_{1,2}^{*}$ represent the adjusted local circulations, and $\Psi_{\mathrm{LS}}$ is the large-scale circulation. The respective surface integrals will also be additive, providing that a domain is selected that is bounded by a contour for which all the $\Psi=0$ and that $\Psi \leq 0$ throughout that domain (which should generally be possible for a sufficiently smooth system). This additivity is important because it means that the partitioning of work in Eq. (3) can also be extended to the components of the coupled two-region system. For example,

$$
W_{T d S}=\iint \Psi_{1}^{*} d S d T+\iint \Psi_{2}^{*} d S d T+\iint \Psi_{\mathrm{LS}} d S d T
$$

Since the surface integrals are additive, a calculation of these quantities using contour integrals will also be additive. An example of this partitioning can be seen in Fig. 3.

As has been mentioned, these integrals are calculated for the entire system using weighted trajectories along contours of the streamfunction in $\left(\theta_{e}, z\right)$ space and with averaged values for the other quantities required for the calculation. The values are calculated separately for each region, while for the large-scale circulation, a mean value is taken, weighted by the contribution of each region to that point in the combined thermodynamic space. As a result of this, the calculation of the largescale terms is more approximate.

\section{c. Anelastic case}

The derivation of Eq. (3) uses the hydrostatic approximation for the reference profile, where the total pressure of all gases in the atmosphere (i.e., dry air and water vapor) is related to height. In the case of an anelastic model, such as the Met Office's large-eddy model (LEM), which is used to derive the numerical results in this paper, it is the partial pressure of dry air that is assumed to be in hydrostatic equilibrium in the reference profile, and as will be seen, the balance of Eq. (3) can be improved by a small correction that is conveniently included in the water vapor component of the Gibbs penalty term and is derived in the appendix. 


\section{Model description and results}

\section{a. Model setup}

The numerical simulations in this section have been produced using a configuration of version 2.4 of the Met Office's LEM in cloud-resolving mode amended to investigate the effect of coupling two regions via the WTG in an idealized context. The underlying LEM is described in Gray et al. (2004), and more complete details of the configuration used here can be found in Daleu et al. (2012). The microphysics are represented by a fivecategory prognostic scheme, with mixing ratios for cloud water, rain, ice, graupel, and snow and with number concentrations for ice, graupel, and snow. Each region is modeled in a two-dimensional configuration with a width of $128 \mathrm{~km}$ (resolution of $500 \mathrm{~m}$ ) and a height of $20 \mathrm{~km}$ (60 vertical levels). To isolate the effect of the coupling strength, a fixed tropospheric cooling profile is used rather than interactive radiation. The cooling rate is fixed at $1.5 \mathrm{~K} \mathrm{day}^{-1}$ below $220 \mathrm{hPa}$ and decreases linearly with pressure to $0 \mathrm{Kday}^{-1}$ at $120 \mathrm{hPa}$, corresponding to integrated atmospheric cooling close to $150 \mathrm{Wm}^{-2}$, depending on the precise value of the surface pressure. The subgrid model is parameterized using a modified first-order Smagorinsky-Lilly approach. For consistency with previous studies with this model, a mixing length of $250 \mathrm{~m}$ was used, but the impact of this choice on the conclusions of this paper was tested and found to be minor.

The WTG velocity is specified by the region-mean potential temperature difference between the two regions and is used to perform advection of both temperature and moisture between the two regions. The two regions are of equal size, and the temperature difference at the surface between them $\Delta T_{s}$, unless otherwise specified, is set to $2 \mathrm{~K}(302.7$ and $304.7 \mathrm{~K}$ for each region). The LEM is run to equilibrium for a 120 -day duration (although no diurnal cycle is modeled), with the first 20 days discarded from the analysis to avoid any transient features, and results are obtained every $30 \mathrm{~min}$, giving 4800 samples of the required variables.

The LEM includes a considerable number of approximations, particularly with respect to the thermodynamics of the system. For example, the specific heat capacities of liquid water and water vapor are taken to be equal (and effectively zero), and the specific enthalpy of water vapor $L_{v}$ does not depend on temperature; these are not consistent with the more exact approach underlying Eq. (3) that is implemented in the diagnostics. Although these approximations are not ideal, the application of a more sophisticated diagnostic framework to motions and variable values derived from a
TABLE 1. Numerical values ( $\mathrm{W} \mathrm{m}^{-2}$ ) for the terms of Eq. (3) for the control case of RCE in a single region: $\Delta \mathscr{G}$ is the unmodified Gibbs penalty component and $\Delta$ is the corresponding discrepancy in the equation; $\Delta \mathscr{G}^{*}$ and $\Delta^{*}$ are the same quantities including the adjustment described in the appendix.

\begin{tabular}{lccccccc}
\hline \hline & $W_{T d S}$ & $W_{b}$ & $W_{p}$ & $\Delta \mathscr{G}$ & $\Delta$ & $\Delta \mathscr{G}^{*}$ & $\Delta^{*}$ \\
\hline Base case & 11.17 & 2.18 & 3.14 & 6.32 & -0.47 & 5.81 & 0.04 \\
Pauluis (2016) & 8.3 & 1.5 & 2.5 & 4.6 & -0.3 & - & - \\
\hline
\end{tabular}

simplified model still provides insight into the full thermodynamic processes that are approximated in the LEM. This choice of model configuration involves a number of compromises that facilitate multiple long simulations over a more detailed representation of the features being modeled, consistent with the idealized nature of the experiments described.

\section{b. Results for single region}

The model was first run for an uncoupled single region over a 302.7-K sea surface temperature. The resulting thermodynamic diagrams (Fig. 2) have already been mentioned. Values for the work terms can be seen in Table 1, in which the effect of the modification described in the appendix is also identified. For comparison, the reference case values calculated in Pauluis (2016; Table 1 herein) are also shown.

It will be seen that the values obtained here are in general some 30\% higher than those in Pauluis (2016). Comparison of the $W_{p}$ thermodynamic diagrams [Fig. $2 \mathrm{~b}$ in this paper and Fig. 4c in Pauluis (2016)] indicates that the atmosphere modeled here has a significantly higher total water mixing ratio, partially explained by the choice of model parameters to represent conditions in the western Pacific-for example, the SST for these simulations is $2.2 \mathrm{~K}$ higher than in Pauluis (2016). Likewise, the $W_{T d S}$ diagram [Fig. $2 \mathrm{~d}$ in this paper and Fig. 4a in Pauluis (2016)] shows higher values for entropy and slightly higher temperatures. The relative values of the mechanical work components are consistent between the two models and consistent with scaling arguments that are developed below.

\section{c. Dependency of components of work done on strength of coupling}

To investigate the impact of the coupling on the generation of mechanical work in the two regions, simulations were performed with values of the WTG timescale $\tau$ between 1 and $50 \mathrm{~h}$; results for two uncoupled regions are also shown for comparison $(\tau=\infty)$. It will be seen that values of the components of work done for the total system remain broadly constant (Fig. 4a), although the introduction of coupling produces a marginal 


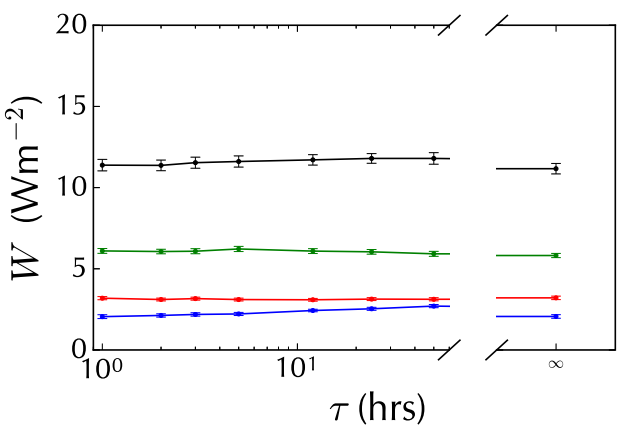

(a) Total system

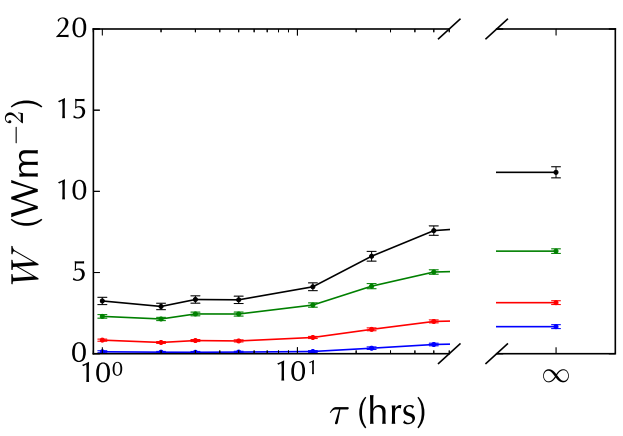

(c) Cooler column

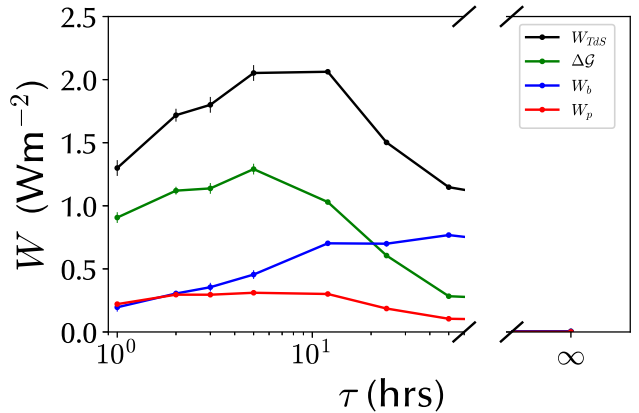

(b) Large-scale circulation

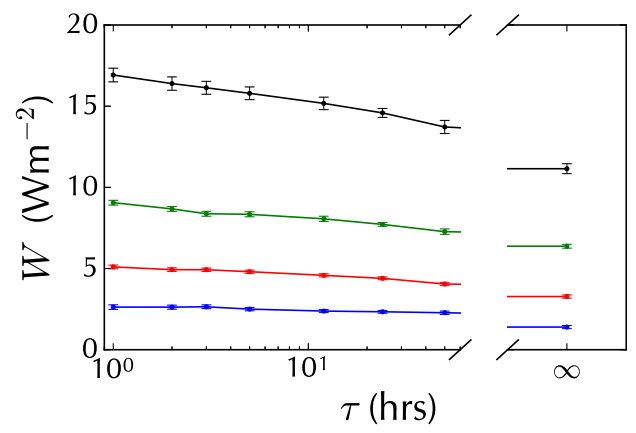

(d) Warmer column

FIG. 4. Components of Eq. (3) for (a) the total system, (b) the large-scale circulation, and the convective circulations in the (c) cooler and (d) warmer regions for varying coupling strengths. A high value of the coupling time-scale parameter $\tau$ indicates weak coupling. The blue line indicates the buoyancy work done $W_{b}$, the red line indicates the moisture elevator term $W_{p}$, the green line indicates the Gibbs penalty $\Delta G^{*}$, and the black line indicates the Carnot maximum $W_{T d s}$. Error bars represent the standard error in the estimate of the parameter, obtained by subsampling.

increase in $W_{b}$ and $W_{T d S}$, which then decreases as the coupling increases.

Values for the local circulations in each of the regions (Figs. $4 \mathrm{c}, \mathrm{d}$ ) on the other hand exhibit a significant difference from the overall mean. This difference is already marked at very weak coupling and increases as the value of $\tau$ decreases, although the values for the cooler region remain almost constant for $\tau<10 \mathrm{~h}$, possibly reflecting the fact that the region approaches a humidity minimum (Fig. 5). Column-integrated water vapor in that region decreases significantly between $\tau=50$ and $10 \mathrm{~h}$ and then shows less variation, indeed a slight increase for $\tau<5 \mathrm{~h}$. As the cooler region dries, the relative importance of the Gibbs penalty component will increase. Weaker convection is associated with a disproportionate decrease in the buoyancy component, as discussed in Pauluis (2016). Figure 6 shows the vertical mass flux for the two regions for $\tau=50 \mathrm{~h}$, which confirms that convection in the cooler region is markedly suppressed even for such weak coupling.

The large-scale circulation (Fig. 4b) shows a different pattern; this includes both regions, which maintain a temperature difference, and hence, $W_{b}$ plays a more prominent role, reducing as the coupling increases and the temperature difference decreases. This decrease is partly compensated by a slight increase in $W_{p}$ as a stronger circulation lifts more moisture. The Gibbs penalty increases with the coupling up to a maximum at around $\tau=5-10 \mathrm{~h}$ before decreasing; this can be attributed to increasing coupling first leading to drying of the cooler region and hence to a decrease in the relative humidity at which energy enters the system (see the scaling arguments below for how this impacts $\Delta \mathscr{G}$ nonlinearly). As discussed above, as $\tau$ decreases below $5 \mathrm{~h}$, the cooler region marginally moistens, which contributes to the increase in $\Delta \mathscr{G}$. The mechanical work terms for components of the large-scale circulation for strong coupling are similar in magnitude to those for the localized circulation in the cooler region; both are substantially smaller than those for the warmer region. As previously mentioned, the balance in the large-scale circulation is more approximate than for the other components and in fact is not improved by inclusion of the correction term described in the appendix. 


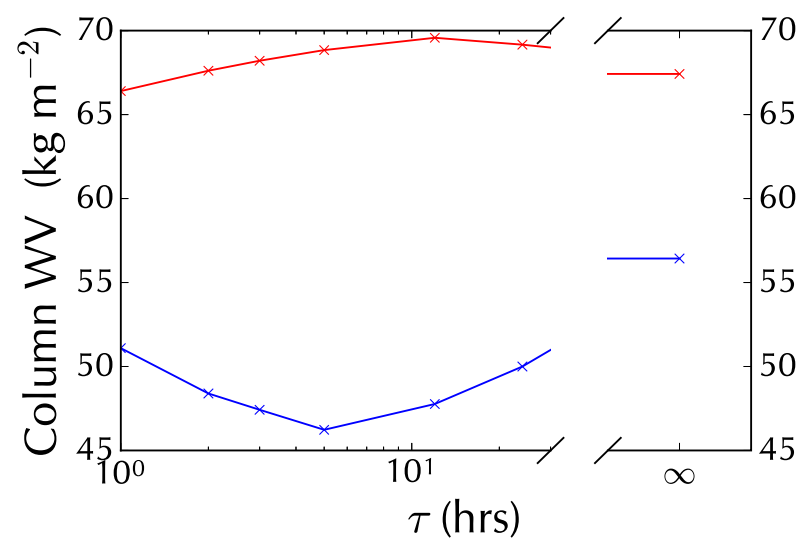

FIG. 5. Integrated column water vapor in the cool region (blue) and warm region (red) for varying values of WTG coupling relaxation time scale $\tau$ for an SST contrast of $2 \mathrm{~K}$.

\section{d. Components of kinetic energy}

The relationship between the strength of the coupling and components of kinetic energy in the total system is shown in Fig. 7. The coupling has an insignificant effect on the kinetic energy in the aggregate system but a strong influence on the vertical kinetic energy associated with the large-scale circulation, which increases with coupling strength. The increase in vertical kinetic energy for smaller $\tau$ indicates that the effect of reducing $\tau$ in increasing $w$ in Eq. (1) is stronger than the effect that enhanced coupling has on reducing the temperature contrast $\theta^{\prime}$ between the regions.

A similar comparison between large-scale and convective horizontal components of kinetic energy would require further assumptions as to the distance between the two regions and their geometry. From a thermodynamic perspective, horizontal motion is of less relevance to the generation of mechanical energy, as it traverses only a small temperature contrast (as forced by the coupling). It is true to say that the horizontal nearsurface flow will likely contribute substantially to the total frictional dissipation in the real system, but in our modeled system, this dissipation is constrained to occur in the two regions.

This comparison between large-scale and convective vertical kinetic energies indicates how weak the largescale circulation is when compared with convective motions. Despite this, the introduction of a large-scale circulation through WTG coupling has very noticeable effects on the strength and nature of the convection within the two regions. In the absence of this large-scale circulation, the mechanical energy budgets in the two regions are very similar. However, even with a very weak large-scale overturning circulation, convection in the warm region is markedly enhanced and that in the cool region suppressed.

\section{e. Dependence on SST difference between the regions}

A further set of numerical experiments was performed for strong coupling $(\tau=2 \mathrm{~h})$, where the temperature difference between the two regions was varied between 0 and $2 \mathrm{~K}$. In each experiment, the regions were of equal area and the mean SST of the aggregate system was $303.7 \mathrm{~K}$. (Plots of the components of the mechanical budget are shown in Fig. 9.) As in the experiment with varying coupling strength, the values of the four components of the mechanical work budget remain broadly constant for the system in aggregate, independent of the SST difference. The values for the cool and warm regions coincide for $\Delta T_{s}=0$ and then diverge gradually. The components of the budget for the large-scale circulation exhibit a quadratic dependency on $\Delta T_{s}$ (red for low values of $\Delta T_{s}$; see also the log plot in Fig. 10), which is discussed below.

\section{Analytical expressions and scaling}

\section{a. Energy conversion under the weak temperature gradient approximation}

The simple form of the WTG means that it is possible to derive an analytical expression for the rate of conversion of potential energy into kinetic energy that it implies. Following Romps (2012), one can express the thermodynamic equation for a one-dimensional WTG system in height coordinates as follows:

$$
\frac{\partial B}{\partial t}=-N^{2} w+Q,
$$

where $B=g \theta^{\prime} / \theta$ is the dry buoyancy, $N$ is the local Brunt-Väisälä frequency $\left[N^{2}=-(g / \theta)(\partial \theta / \partial z)\right]$, and $Q$ is the external heat input into the system. It is assumed that $N$ does not have any dependency on time although it can vary with height.

If the WTG approximation is used, the vertical velocity (above the boundary layer) can be expressed as

$$
w=\frac{\theta^{\prime}}{\tau \partial \theta / \partial z}=\frac{B}{\tau N^{2}} .
$$

The available potential energy (APE) introduced by Lorenz (1960) $\bar{A}$ of the system can be described locally in terms of these variables by dry "availability": $A=B^{2} / 2 N^{2}$. It can be shown that the density-weighted column integral of $A$ is equal to $\bar{A}$. The time derivative of the local availability is given by

$$
\frac{\partial A}{\partial t}=\frac{2 B}{2 N^{2}} \frac{\partial B}{\partial t}=\frac{B Q}{N^{2}}-B w=\frac{B Q}{N^{2}}-\frac{2 A}{\tau} .
$$




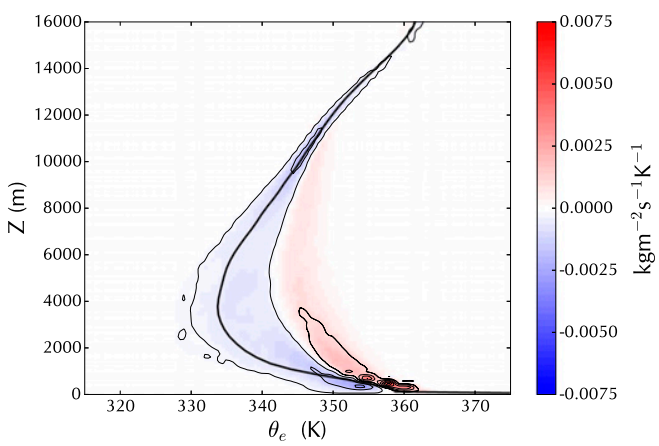

(a) Cooler column

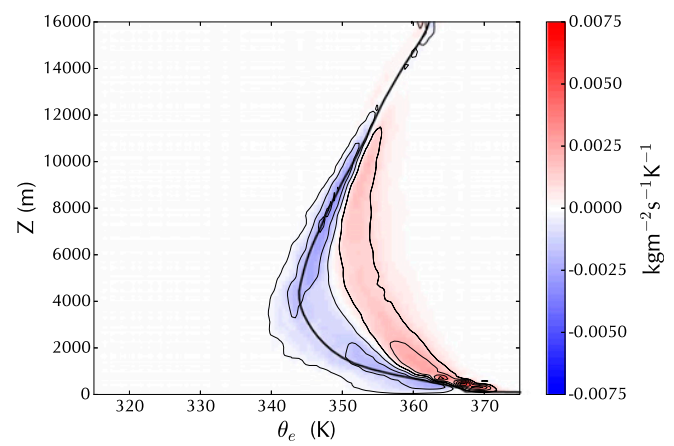

(b) Warmer column

FIG. 6. Isentropic distribution of the vertical mass flux $\langle\rho w\rangle$ for (a) the cool region and (b) the warm region with coupling $\tau=50 \mathrm{~h}$. The solid curve represents the mean profile of the equivalent potential temperature $\theta_{e}$.

This can be interpreted as indicating that availability is increased by a differential heating term $B Q / N^{2}$, which is balanced by a conversion term from potential to kinetic energy of

$$
C_{A \rightarrow \mathrm{KE}}=\frac{2 A}{\tau} .
$$

Thus, for the system as a whole (ignoring the complication of the boundary layer interpolation), the conversion to kinetic energy will be $2 \bar{A} / \tau$. It will also be noted that Eq. (10) requires heat input to correlate with positive buoyancy, which is consistent with the second law of thermodynamics. The effect of the boundary layer interpolation is to weaken the coupling in the lowest layers, which can be represented by increasing the value of $\tau$ for those layers - the impact of this adjustment is minor.

Figure 8 compares the conversion term from Eq. (11) and the large-scale components of the mechanical energy budget as in Fig. 4b, which of course includes moist variables. The "mechanical work done" term $W_{b}$ scales as $2 \bar{A} / \tau$; in fact, the values coincide. As the APE has a quadratic dependency on the temperature difference between the two regions (which will in turn depend linearly on the temperature difference at the surface), a quadratic dependency of the work terms on $\Delta T_{s}$ may be anticipated. This is indeed seen in Fig. 9b as presented in section $3 \mathrm{e}-\mathrm{a} \log -\log$ plot of the same values is shown in Fig. 10 .

\section{b. Scaling for components of work done}

Further scalings can be developed for the relative values of the terms in the mechanical energy budget. These do not depend on the assumption of a coupling mechanism. In an anelastic model such as the LEM, the buoyancy [see Gray et al. 2004, their Eqs. (11)-(13)] is calculated as

$$
B=g\left[\frac{\theta^{\prime}}{\theta_{r}}+\left(\frac{R_{v}}{R_{d}}-1\right) r_{v}-\sum_{w \neq v} r_{w}\right],
$$

where $R_{v}$ and $R_{d}$ are the specific gas constants for water vapor and dry air and $\theta_{r}$ is the reference-state potential temperature. Hence, if we assume that variations in specific humidity dominate the buoyancy rather than any temperature differences as is suggested by these model results, and also that $\delta r_{T} \approx \delta r_{v}$, only the second term in Eq. (12) need be considered for scaling purposes. Hence, the buoyancy lifting term, $W_{b}$ in Eq. (3), will scale as $\left(R_{v} / R_{d}-1\right) W_{p}=0.6 W_{p}$, with $W_{p}$ being the moisture lifting term. The approximate scaling between $W_{b}$ and $W_{p}$ can be readily verified from the numerical

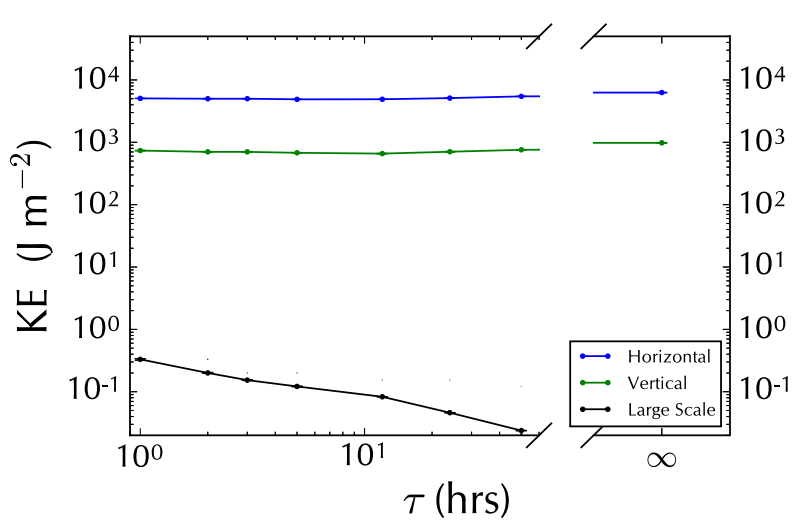

FIG. 7. Components of kinetic energy. The blue line represents the horizontal component and the green line the vertical component for the aggregate system. The contribution of the large-scale motion to the vertical kinetic energy is shown in black. 
values shown in Table 1 and also holds for the warmer region for the two-region system (Fig. 4d), which dominates the aggregate system (Fig. 4a). In circumstances where the temperature differences are significant (and correlated with moisture anomalies), such as for the large-scale circulation at low coupling strength, one could expect $W_{b} / W_{p}$ to have a value greater than 0.6 as is observed (Fig. 4b).

Pauluis [2011, his Eq. (24)] gives an expression for the Gibbs penalty. Assuming that condensation occurs close to saturation, that vapor anomalies dominate as before, and that the sensible heat component is negligible, an approximate expression for this term can be written in terms of the net rate of evaporation at the surface $\left(E=\Delta r_{v} / \Delta t\right)$ :

$$
\Delta \mathscr{G} \approx-R_{v} T_{\text {out }} E \ln \mathscr{H}_{\text {in }},
$$

where $\mathscr{H}$ is relative humidity and the subscripts "in" and "out" indicate conditional harmonic means over the zones where heat enters and leaves the system. For example, if we write $F_{\text {in }}$ for heat input and $\mathscr{b}$ as the system's boundary, then following Ambaum [2010, his Eq. (10.50)],

$$
\frac{1}{T_{\text {in }}}=\left(\int_{\mathscr{b}} F_{\text {in }} d \mathscr{b}\right)^{-1} \int_{\mathscr{b}} \frac{F_{\text {in }}}{T} d \mathscr{b} .
$$

Likewise, $T_{\text {out }}$ is defined using $F_{\text {out }}$, the heat output of the system, and $\mathscr{H}_{\text {in }}$ is defined as in Pauluis [2011, his Eq. (16)].

Likewise, $W_{T d S}$ will be mainly driven by the latent heat input and scales with the generalized Carnot efficiency:

$$
\begin{aligned}
W_{T d S} & \approx \frac{T_{\text {in }}-T_{\text {out }}}{T_{\text {in }}}\left(L_{v} E+\Delta \mathscr{G}\right) \\
& \approx \frac{T_{\text {in }}-T_{\text {out }}}{T_{\text {in }}} L_{v} E
\end{aligned}
$$

where $L_{v}$ is the enthalpy of vaporization of water. It will be seen from Eq. (13) that the Gibbs penalty term can be neglected in scaling Eq. (15), given that $R_{v} T_{\text {out }} / L_{v} \approx 0.05$. Eliminating $E$ between Eqs. (13) and (16),

$$
\Delta \mathscr{G} \approx-\frac{R_{v} T_{\text {out }} T_{\text {in }}}{L_{v}\left(T_{\text {in }}-T_{\text {out }}\right)} \ln \mathscr{B}{ }_{\text {in }} W_{T d S} .
$$

Values of $295 \mathrm{~K}$ for $T_{\text {in }}$ and $280 \mathrm{~K}$ for $T_{\text {out }}$ can be obtained from the model (or even by inspection of Fig. 2d, which shows values for the RCE case, which will also be typical for the aggregate system). An average value for $\mathscr{H}_{\text {in }}$ is more problematic to define, but using $60 \%$ is

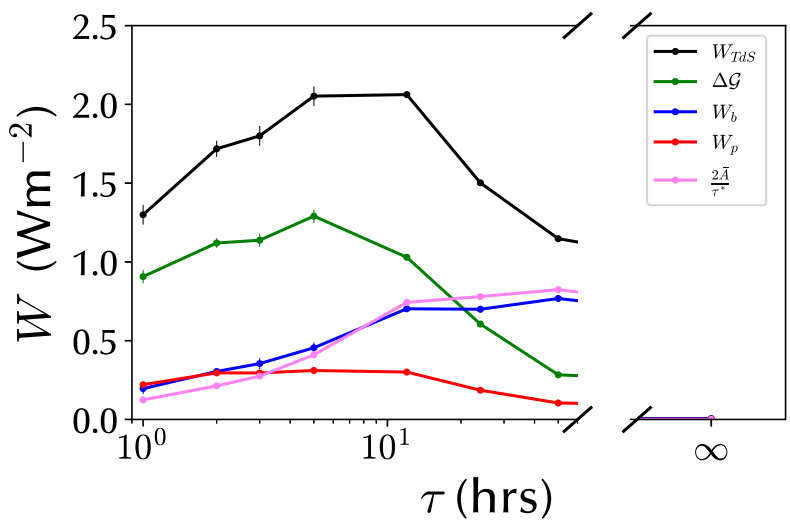

FIG. 8. As in Fig. 4b, but also including the conversion rate from potential to kinetic energy based on Eq. (11) (violet).

consistent with model results and the observed values of $\Delta \mathscr{G} \approx 0.5 W_{T d S}$.

It is possible to develop Eq. (17) further by making use of the Clausius-Clapeyron relation in the form $d e_{s} / e_{s}=L_{v} d T / R_{v} T^{2}$ :

$$
\begin{aligned}
\frac{\Delta \mathscr{G}}{W_{T d S}} & \approx-\frac{R_{v} T_{\text {out }} T_{\text {in }}}{L_{v}\left(T_{\text {in }}-T_{\text {out }}\right)} \ln \mathscr{H}_{\text {in }}, \\
& \approx \frac{\ln \mathscr{H}_{\text {in }}}{\ln \left(\frac{e_{s, \text { out }}}{e_{s, \text { in }}}\right)},
\end{aligned}
$$

where $e_{s, \text { in }}$ and $e_{s, \text { out }}$ are the saturated vapor pressures of water at temperatures $T_{\text {in }}$ and $T_{\text {out }}$. Defining $r_{v \text {,in }}$ as the vapor mixing ratio corresponding to $\mathscr{H}_{\text {in }}$ at temperature $T_{\text {in }}$ and dry pressure $p_{d \text {,in }}$,

$$
\frac{\Delta \mathscr{G}}{W_{T d S}}=\frac{\ln \left(\frac{R_{v} r_{v, \text { in }} p_{d, \text { in }}}{R_{d} e_{s, \text { out }}}\right)+\ln \left(\frac{e_{s, \text { out }}}{e_{s, \text { in }}}\right)}{\ln \left(\frac{e_{s, \text { out }}}{e_{s, \text { in }}}\right)} .
$$

We now introduce $r_{v s, \text { out }}$ via $e_{s, \text { out }}=R_{v} / R_{d} p_{d, \text { out }} r_{v s, \text { out }}$, where $p_{d, \text { out }}$ is the partial pressure of dry air at temperature $T_{\text {out }}$. Substituting for $e_{s, \text { out }}$ in the first logarithm in the numerator of Eq. (20) leads to

$$
\frac{\Delta \mathscr{G}}{W_{T d S}}=1-\frac{\ln \left(\frac{p_{d, \text { out }}}{p_{d, \text { in }}}\right)}{\ln \left(\frac{e_{s, \text { out }}}{e_{s, \text { in }}}\right)}-\frac{\ln \left(\frac{r_{v s, \text { out }}}{r_{v, \text { in }}}\right)}{\ln \left(\frac{e_{s, \text { out }}}{e_{s, \text { in }}}\right)} .
$$

Assuming that $r_{v s, \text { out }} \leqslant r_{v \text {,in }}$ (see Fig. 2b to confirm that this is reasonable for all but the shallowest circulations), 


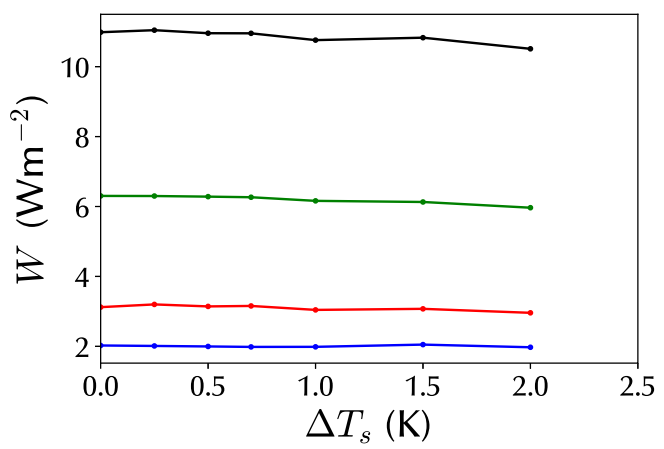

(a) Total system

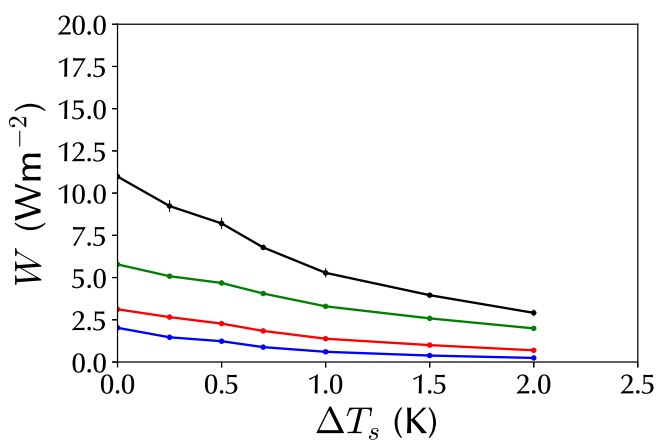

(c) Cooler column

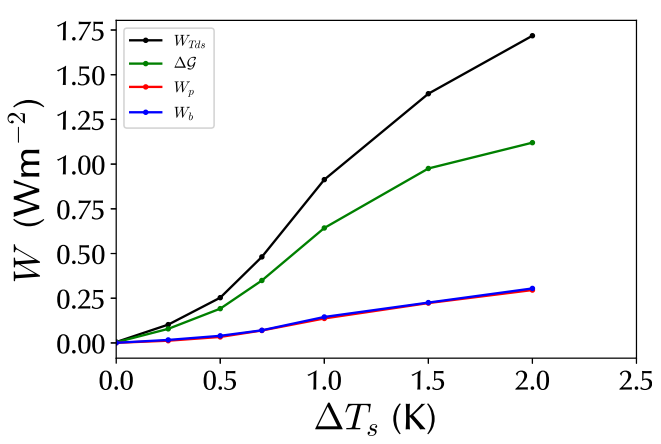

(b) Large-scale circulation

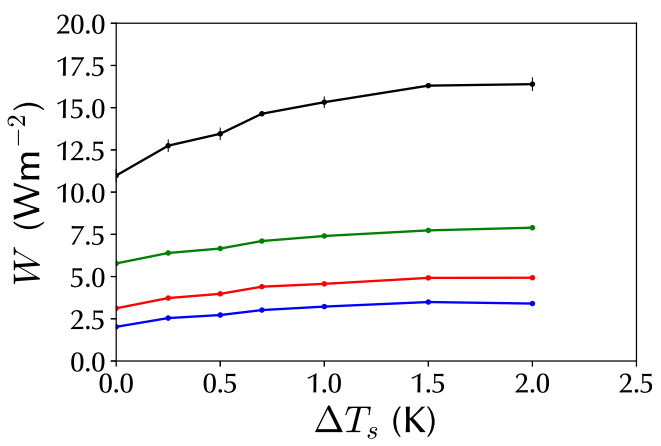

(d) Warmer column

FIG. 9. Components of Eq. (3) for (a) the total system, (b) the large-scale circulation, and the convective circulations in the (c) cooler and (d) warmer regions for varying values of $\Delta T_{s}$, the difference between the SSTs in the two regions. The blue line indicates the buoyancy work done $W_{b}$, the red line indicates the moisture elevator term $W_{p}$, the green line indicates the Gibbs penalty $\Delta G^{*}$, and the black line indicates the Carnot maximum $W_{T d s}$. In (b), the red and blue lines largely coincide.

$$
\frac{\Delta \mathscr{G}}{W_{T d S}} \lesssim 1-\frac{\ln \left(\frac{p_{d, \text { out }}}{p_{d, \text { in }}}\right)}{\ln \left(\frac{e_{s, \text { out }}}{e_{s, \text { in }}}\right)} .
$$

Making a Taylor expansion in $\ln (1+x) \approx x$,

$$
\begin{aligned}
\frac{\Delta \mathscr{G}}{W_{T d S}} & \leq 1-\frac{\Delta z / H_{p d}^{*}}{\Delta z / H_{e s}^{*}}, \\
& \leq 1-\frac{H_{e s}^{*}}{H_{p d}^{*},} \\
& \leq 1-\frac{R_{v} g T}{R_{d} L_{v} \Gamma},
\end{aligned}
$$

where $\Delta z$ is the height difference between the in and out states, $H_{e s}^{*}=-R_{v} \bar{T}^{2} / L_{v} \Gamma$ and $H_{p d}^{*}=R_{d} \bar{T} / g$ are the scale heights for saturated vapor pressure and the partial pressure of dry air, $\bar{T}$ is an average temperature over the cycle between $T_{\text {in }}$ and $T_{\text {out }}$, and $\Gamma$ is the atmospheric lapse rate. Using $\bar{T}=290 \mathrm{~K}$ and $\Gamma=-5 \mathrm{~K} \mathrm{~km}^{-1}$ gives $\Delta \mathscr{G} / W_{T d S}<0.64$. This ratio will decrease as $r_{v, \text { out }} / r_{v \text {, in }}$

decreases (i.e., as the temperature difference between the "in" and "out" states increases).

Combining these various elements as in Eq. (3), one can expect the efficiency with which such a system in aggregate generates mechanical work $W_{b}$ to be typically $20 \%$ of the generalized Carnot maximum $W_{T d S}$. The values shown in Table 1 and in Fig. 4 are consistent with these scalings.

There is an imbalance in latent heat input between the regions, consistent with the transport of moisture from the cool region to the warm region. The Gibbs penalty component of the mechanical energy budget for the large-scale circulation is strongly correlated with this moisture transport between the regions (Fig. 11). The relationship between these two quantities can be explained using Eq. (13), using the net moisture advected by the large-scale circulation:

$$
\Delta \mathscr{G} \approx \frac{-R_{v}}{L_{v}} T_{\text {out }} \ln \mathscr{H}_{\text {in }} \times \text { latent heat transport } .
$$

Using $T_{\text {out }}=285 \mathrm{~K}$ and $\mathscr{H}$ in $=60 \%$ as before provides a good fit. 


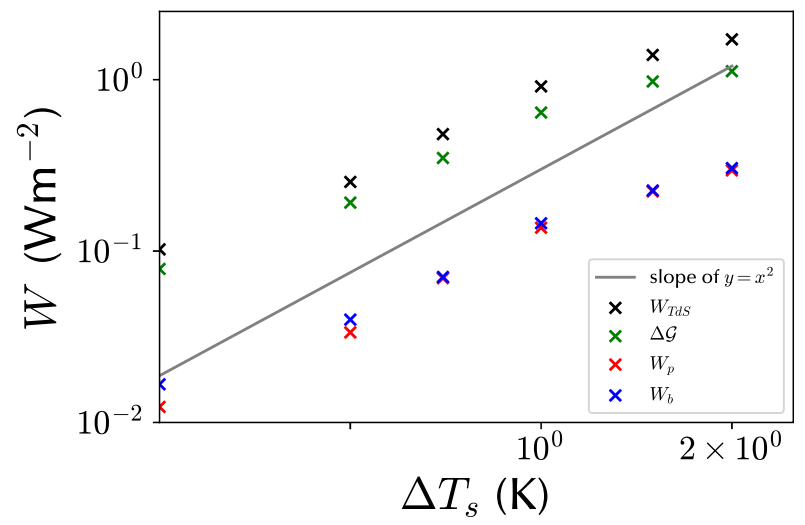

FIG. 10. Components of Eq. (3) for the large-scale circulation (as shown in Fig. 9, but plotted on a log-log scale) for varying values of $\Delta T_{s}$, the difference between the SSTs in the two regions. The blue line indicates the buoyancy work done $W_{b}$, the red indicates the moisture elevator term $W_{p}$, the green line indicates the Gibbs penalty $\Delta G^{*}$, and the black line indicates the Carnot maximum $W_{T d S}$.

\section{Summary and discussion}

This paper shows how the mechanical energy budget for a convecting system proposed in Pauluis (2016) can be applied in the case of two coupled regions, permitting attribution of each of the components to either localized features within one of the two regions or to the large-scale circulation. It also proposes a refinement to the basic approach in the case of an anelastic cloud-resolving model. Our work has not identified any energetic inconsistencies introduced by the use of the WTG approximation as a proxy for largescale circulation.

Numerical results were obtained using a CRM in which two regions are coupled using the WTG approximation. They demonstrate that the terms in the mechanical energy budget of the system considered as a whole are insensitive to the strength (indeed even the presence) of the coupling, indicating that the coupling does not distort the energy balance of the whole system. However, even very weak coupling has a marked effect on the nature of the convection within each region and its associated energy budget terms. As the strength of the coupling increases, the convection in the cool region is further suppressed and that in the warmer region enhanced. It is possible that some of this insensitivity is a consequence of using a fixed cooling profile rather than an interactive radiative model. However, other broad properties of the system appear to be independent of the coupling strength, which suggests that an interactive radiative scheme would be likely to produce similar results. Further numerical experiments show that the mechanical energy budget for the entire system is equally insensitive to changes in the difference in SSTs between the columns.

The ratios between the component terms contributing to the total energy flows for the entire system and for each of the regions (where the effect of the largescale velocity is isolated) are relatively constant as a function of coupling strength and are consistent with estimates obtained by theoretical scaling arguments. On the other hand, the values of the component terms for the large-scale circulation exhibit markedly different features in that the buoyancy contribution is more significant than that due to the lifting of precipitation, while the Gibbs penalty reaches a maximum as the cooler region dries out before then decreasing as the differences between the regions are eliminated with stronger coupling.

The buoyancy contribution to the large-scale circulation is consistent with that predicted from an analytical

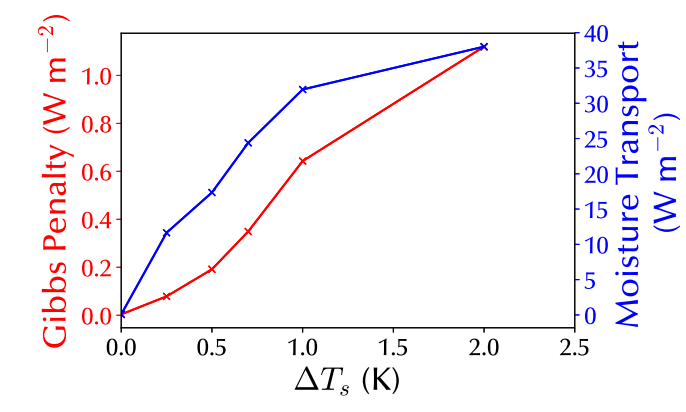

(b) SST difference (a) Coupling strength

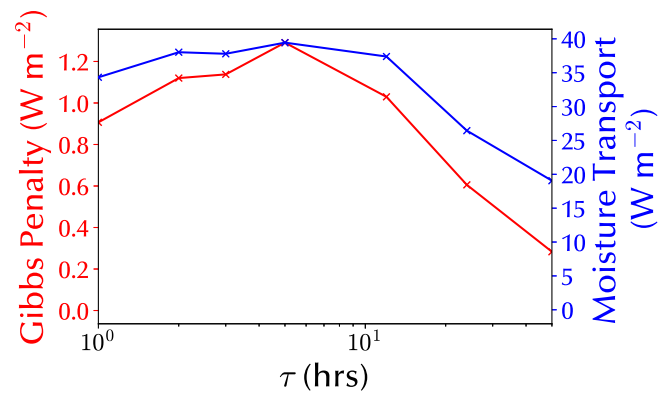

FIG. 11. Comparison between the Gibbs penalty component of the large-scale circulation (red, left-hand axis) and latent heat transported between the regions (blue, right-hand axis) for (a) the numerical experiment where coupling strength is varied and (b) the numerical experiment where SST difference is varied. 
approach to the energetics implied by WTG in a dry setup, which also predicts its quadratic dependency on the SST difference between the columns that is observed. We also present more general scaling arguments for the components of a mechanical energy budget, which could serve to indicate the impact of changing atmospheric conditions on the generation of kinetic energy.

The vertical kinetic energy associated with the largescale circulation is found to be more than three orders of magnitude smaller than the vertical kinetic energy associated with the convection within each region. Despite this weak magnitude, the coupling has a strong influence on the suppression and enhancement of convection in each of the regions. The imposition of the WTG approximation may be interpreted as a representation of the requirement for low temperature gradients in the tropics due to weak rotational effects. In this sense, the contrast in local convective intensity can be seen as the consequence of a large-scale dynamical constraint and not as a local result of contrast in SSTs.

The techniques introduced in this paper, suitably modified, can be applied to reanalysis products to estimate a mechanical work budget for large-scale circulations such as the Walker or Hadley circulations, which would provide further insight into whether coupling techniques such as the WTG provide a useful approach to reflecting such phenomena in studies of convection. Similarly, they could shed light on the mechanics of convective aggregation, which can be studied using the WTG approximation (Emanuel et al. 2014).

Acknowledgments. Jan Kamieniecki is supported by a grant from the U.K. Natural Environment Research Council (Grant NE/K004034/1). We thank the Met Office for access to version 2.4 of the LEM, C. L. Daleu for making her model configuration available to us, and David Raymond, Timothy W. Cronin, and one anonymous reviewer for their helpful comments.

\section{APPENDIX}

\section{Components of Work Done in an Anelastic Model}

In this appendix, we explain how the derivation of the components of work [Eq. (3)] in Pauluis (2016) can be refined in the case of an anelastic model, and we derive an approximate expression for a suggested correction term.

\section{a. Hydrostatic balance in an anelastic context}

The original derivation of Eq. (3) involves the use of the hydrostatic approximation $d p=-\rho_{\text {ref }} g d z$ in evaluating $-\alpha_{d} d p$, where $\rho_{\text {ref }}$ is the density of dry air in the reference profile and $\alpha_{d}$ is the specific volume of dry air. In an anelastic model such as the LEM, this need not hold in general, but it does hold for the reference profile, where it relates the partial pressure of dry air $p_{0}$ to height: $d p_{0}=-\rho_{\text {ref }} g d z$.

\section{b. Derivation of approximate correction term}

The original derivation of Eq. (3) can be restated as below:

$$
\oint T d s=-\oint \alpha_{d} d p-\sum_{w=v, l, i} \oint g_{w} d r_{w}
$$

where

$$
\begin{aligned}
\alpha_{d} & =\frac{R_{d} T+r_{v} R_{v} T}{p} \\
& \approx \frac{R_{d} T_{\text {ref }}}{p}\left[1+\frac{T^{\prime}+r_{v}\left(R_{v} / R_{d}\right) T_{\text {ref }}-r_{T} T_{\text {ref }}}{T_{\text {ref }}}+r_{T}\right] \\
& =\frac{R_{d} T_{\text {ref }}}{p}\left(1+\frac{B}{g}+r_{T}\right),
\end{aligned}
$$

using the definition of $B$ in Eq. (12) and where $R_{d}$ and $R_{v}$ are the specific gas constants for dry air and water vapor, respectively, and $T_{\text {ref }}$ the temperature of the reference profile, ignoring expressions that are second order in the mixing ratio or buoyancy. Likewise, one can then approximate the contour integrals of the second and third terms on the right-hand side of Eq. (A4):

$$
\begin{aligned}
& -\oint \frac{R_{d} T_{\mathrm{ref}} B}{g p} d p \approx-\oint \frac{R_{d} T_{\mathrm{ref}} B}{g p_{0}} d p_{0}=\oint B d z \\
& -\oint \frac{R_{d} T_{\mathrm{ref}} r_{T}}{p} d p \approx \oint g r_{T} d z .
\end{aligned}
$$

The contour integral of the first term on the right-hand side of Eq. (A4) is zero for an anelastic model if the full hydrostatic approximation is used as in Pauluis (2016). Applying instead the hydrostatic approximation for dry air, it can be simplified with the assumption that the reference profile is close to the equilibrium state and that variations in the pressure of dry air at a given height are insignificant when compared with variations in water vapor pressure, (i.e., $p \approx p_{\text {ref }}+e$ ) and ignoring higher orders of $e / p_{\text {ref }}$ to obtain an additional term:

$$
-\oint \frac{R_{d} T_{\text {ref }}}{p} d p=-\oint \frac{R_{d} T_{\text {ref }}}{p_{\text {ref }}+e} d\left(p_{\text {ref }}+e\right)
$$




$$
\begin{aligned}
\approx & -\oint \frac{R_{d} T_{\text {ref }}}{p_{\text {ref }}} d p_{\text {ref }}+\oint \frac{e R_{d} T_{\text {ref }}}{p_{\text {ref }}^{2}} d p_{\text {ref }} \\
& -\oint \frac{R_{d} T_{\text {ref }}}{p_{\text {ref }}} d e .
\end{aligned}
$$

Making use of the gas law,

$$
\begin{aligned}
-\oint \frac{R_{d} T_{\mathrm{ref}}}{p} d p \approx & -\oint \frac{1}{\rho_{\mathrm{ref}}} d p_{\text {ref }}+\oint \frac{e R_{d} T_{\mathrm{ref}}}{p_{\mathrm{ref}}^{2}} d p_{\mathrm{ref}} \\
& -\oint \frac{R_{d} T_{\mathrm{ref}}}{p_{\mathrm{ref}}} d e .
\end{aligned}
$$

Using the hydrostatic approximation and remembering that $e=r_{v}\left(R_{v} / R_{d}\right) p_{\text {ref }}$

$$
\begin{aligned}
-\oint \frac{R_{d} T_{\text {ref }}}{p} d p \approx & \oint g d z+\oint \frac{r_{v} R_{v} T_{\text {ref }}}{p_{\text {ref }}} d p_{\text {ref }}-\oint \frac{r_{v} R_{v} T_{\text {ref }}}{p_{\text {ref }}} d p_{\text {ref }} \\
& -\oint R_{v} T_{\text {ref }} \frac{d r_{v}}{d p_{\text {ref }}} d p_{\text {ref }} .
\end{aligned}
$$

The first of the terms on the right-hand side is zero, and the second and third cancel, leaving the final term, which can be expressed as an integral in $r_{v}$ :

$$
-\oint \frac{R_{d} T_{\mathrm{ref}}}{p} d p \approx-\oint R_{v} T_{\mathrm{ref}} d r_{v} .
$$

This expression can be integrated by parts and then expressed in terms of an integral in $z$ :

$-\oint \frac{R_{d} T_{\mathrm{ref}}}{p} d p \approx+\oint R_{v} r_{v} d T_{\mathrm{ref}}=-\oint R_{v} r_{v} \Gamma d z$

where $\Gamma$ is the lapse rate of the reference profile. Equation (A11) is the form of the equation that is used to calculate this term numerically in the contour integrals; Eq. (A12) provides a more useful form in developing scaling arguments below.

The new term in the form shown in Eq. (A11) bears a similarity to a term in the water vapor component of the Gibbs penalty $\oint\left(-\mathscr{G}_{v}\right) d r_{v}$, depending as it does on the same state variables since

$$
\mathscr{G}_{v}=c_{l}\left(T-T_{f}-T \ln \frac{T}{T_{f}}\right)+R_{v} T \ln \mathscr{H},
$$

where $c_{l}$ is the specific heat capacity of liquid water at constant pressure, $T_{f}$ its freezing temperature, and $\mathscr{H}$ the relative humidity. One can therefore introduce a modified specific Gibbs free energy for the vapor state:

$$
\mathscr{G}_{v}^{*}=c_{l}\left(T-T_{f}-T \ln \frac{T}{T_{f}}\right)+R_{v} T(1+\ln \mathscr{H}),
$$

and (neglecting a term in $T_{\text {ref }}-T$ ) instead of Eq. (3), one can write

$\oint T d S \approx \oint B d z+\oint r_{T} g d z+\sum_{w=v, l, i} \oint\left(-\mathscr{G}_{w}^{*}\right) d r_{w}$,

where $\mathscr{G}_{w}^{*}=\mathscr{G}_{w}$ for $w=l, i$. (It could be argued that from a physical perspective this term is a correction to the mechanical work generated and should therefore instead be combined with $W_{b}$.)

\section{c. Numerical values and scalings}

The contribution of this new term can be illustrated by comparing numerical values for the components of Eq. (A15) with the modified version produced by the LEM for the case of a single uncoupled convecting region in a state of equilibrium over a surface at a temperature of $302.7 \mathrm{~K}$ (as discussed in section $3 \mathrm{~b}$ ). As Table 1 shows, the effect of the correction term is to reduce the discrepancy in the equation from around $5 \%$ of the largest term to less than $1 \%$. Similar impacts are observed in other configurations of the model, including in the coupled version.

The additional term expressed in the form in Eq. (A12) will scale as approximately $20 \%$ of $W_{p}$ in Eq. (3), for a moist adiabatic temperature profile where the lapse rate of the reference state $\Gamma$ will be approximately half the dry adiabatic lapse rate $g / c_{p}$, where $c_{p}$ is the specific heat capacity of dry air at constant pressure given that $R_{v} / c_{p}=0.46$.

\section{REFERENCES}

Ambaum, M. H. P., 2010: Thermal Physics of the Atmosphere. John Wiley and Sons, $252 \mathrm{pp}$.

Daleu, C. L., S. J. Woolnough, and R. S. Plant, 2012: Cloudresolving model simulations with one- and two-way couplings via the weak temperature gradient approximation. J. Atmos. Sci., 69, 3683-3699, https://doi.org/10.1175/JAS-D-12-058.1.

,$- \ldots$, and $-2015 \mathrm{a}$ : Transition from suppressed to active convection modulated by a weak temperature gradientderived large-scale circulation. J. Atmos. Sci., 72, 834-853, https://doi.org/10.1175/JAS-D-14-0041.1.

_ _ , and Coauthors, 2015b: Intercomparison of methods of coupling between convection and large-scale circulation: 1. Comparison over uniform surface conditions. J. Adv. Model. Earth Syst., 7, 1576-1601, https://doi.org/10.1002/ 2015MS000468.

__, and Coauthors, 2016: Intercomparison of methods of coupling between convection and large-scale circulation: 2. Comparison over nonuniform surface conditions. $J . A d v$. Model. Earth Syst., 8, 387-405, https://doi.org/10.1002/ 2015MS000570. 
Emanuel, K. A., 1986: An air-sea interaction theory for tropical cyclones. Part I: Steady-state maintenance. J. Atmos. Sci., 43, 585-605, https://doi.org/10.1175/1520-0469(1986)043<0585: AASITF $>2.0 . \mathrm{CO} ; 2$

_ A. A. Wing, and E. M. Vincent, 2014: Radiative-convective instability. J. Adv. Model. Earth Syst., 6, 75-90, https://doi.org/ 10.1002/2013MS000270.

Gray, M. E. B., J. Petch, S. H. Derbyshire, A. R. Brown, A. P. Lock, H. A. Swann, and P. R. A. Brown, 2004: Version 2.3 of the Met Office large eddy model: Part II. Scientific documentation. Met Office Rep., 49 pp.

Herman, M. J., and D. J. Raymond, 2014: WTG cloud modeling with spectral decomposition of heating. J. Adv. Model. Earth Syst., 6, 1121-1140, https://doi.org/10.1002/2014MS000359.

Kuang, Z., 2008: Modeling the interaction between cumulus convection and linear gravity waves using a limited-domain cloud system-resolving model. J. Atmos. Sci., 65, 576-591, https:// doi.org/10.1175/2007JAS2399.1.

Lorenz, E. N., 1960: Generation of available potential energy and the intensity of the general circulation. Dynamics of Climate, R. C. Pfeffer, Ed., Pergamon Press, 86-92.

Pauluis, O., 2011: Water vapor and mechanical work: A comparison of Carnot and steam cycles. J. Atmos. Sci., 68, 91-102, https://doi.org/10.1175/2010JAS3530.1.

_, 2016: The mean air flow as Lagrangian dynamics approximation and its application to moist convection. J. Atmos. Sci., 73, 4407-4425, https://doi.org/10.1175/JAS-D-15-0284.1.
— , and I. M. Held, 2002a: Entropy budget of an atmosphere in radiative-convective equilibrium. Part I: Maximum work and frictional dissipation. J. Atmos. Sci., 59, 125-139, https:// doi.org/10.1175/1520-0469(2002)059<0125:EBOAAI > 2.0.CO;2.

- and —, 2002b: Entropy budget of an atmosphere in radiative-convective equilibrium. Part II: Latent heat transport and moist processes. J. Atmos. Sci., 59, 140-149, https:// doi.org/10.1175/1520-0469(2002)059<0140:EBOAAI > 2.0.CO;2

— and A. A. Mrowiec, 2013: Isentropic analysis of convective motions. J. Atmos. Sci., 70, 3673-3688, https://doi.org/10.1175/ JAS-D-12-0205.1.

— V. Balaji, and I. M. Held, 2000: Frictional dissipation in a precipitating atmosphere. J. Atmos. Sci., 57, 989-994, https:// doi.org/10.1175/1520-0469(2000)057<0989:FDIAPA>2.0.CO;2.

Raymond, D. J., and X. Zeng, 2005: Modelling tropical atmospheric convection in the context of the weak temperature gradient approximation. Quart. J. Roy. Meteor. Soc., 131, 1301-1320, https://doi.org/10.1256/qj.03.97.

Romps, D. M., 2012: Weak pressure gradient approximation and its analytical solutions. J. Atmos. Sci., 69, 2835-2845, https:// doi.org/10.1175/JAS-D-11-0336.1.

Sobel, A. H., and C. S. Bretherton, 2000: Modeling tropical precipitation in a single column. J. Climate, 13, 4378-4392, https:// doi.org/10.1175/1520-0442(2000)013<4378:MTPIAS> 2.0.CO;2 